\title{
Narración.
}

\section{Sobre Nuestra ciudad de Thornton Wilder} Narration.

\section{About Thornton Wilder's Our City}

\section{Antonio Lastra}

(D) https://orcid.org/0000-0002-4470-4494

Instituto Franklin de Investigación en Estudios Norteamericanos, Universidad de Alcalá, España.

antoniolastra@latorredelvirrey.es

\section{Resumen}

Hasta qué punto Nuestra ciudad depende de las enseñanzas de Gertrude Stein, a quien Thornton Wilder se refería como su "Diótima", es una de las entradas más difíciles, y más compensatorias, de los estudios sobre la obra. Se ha hablado de influencia y de catálisis, de anticipación y coincidencia. Wilder había ensayado en muchas de sus obras en un solo acto, previas a Nuestra ciudad, la renuncia a la trama y la acción dramática, así como el despojamiento del escenario -como había hecho Stein en sus propias obras de teatro-, pero aún había de encontrar la forma definitiva para lo que quería decir. Diez años después de El puente de San Luis Rey, y a pesar de que seguía trabajando en $E /$ cielo, mi destino, esa forma definitiva tenía que permitirle desaparecer como autor de novelas para que la vida cotidiana, sobre la que no cabe la omnisciencia, ocupara el lugar que le correspondía.

Palabras clave: Estudios Culturales, narración, Gertrude Stein, influencia, forma literaria.

\section{Abstract}

To what extent Our citydepends on the teachings of Gertrude Stein, whom Thornton Wilder referred to as his "Diotima", is one of the most difficult, and most compensatory, entries in the studies on the work. It has been talk of influence and catalysis, anticipation and coincidence. Wilder had rehearsed in many of his plays in a single act prior to Our City there nunciation of plot and dramaticaction, as well as the stripping of the stage -as Stein herselfhad done in hisownplays - but he had yet to find the final form for what he crave to say. Ten years after The Bridge of San Luis Rey, and despite the fact that he was still working on Heaven, my destination, that definitive form had to allow him to disappear as the author of novels so that daily life, about which there is no room for omniscience, would occupy the his rightful place.

Key words: Cultural Studies, narration, Gertrude Stein, influence, literary form.

Sumario

1. Clásico y comercial | 2. La escritura dramática | 3. Narración | Referencias bibliográficas

\section{Cómo citar este artículo}

Lastra, A. (2020): "Narración. Sobre Nuestra ciudad de Thornton Wilder", methaodos. revista de ciencias sociales, 8 (1): 112-119. http://dx.doi.org/10.17502/m.rcs.v8i1.344 


\section{Clásico y comercial ${ }^{1}$}

En su libro de homenaje al escritor William Somerset Maugham, Garson Kanin, escritor él mismo y cineasta, registró una visita al crítico literario Edmund Wilson en su casa de Provincetown (Massachusetts), en el verano de 1960, acompañado por su mujer, la actriz y guionista Ruth Gordon, y su amigo y maestro Thornton Wilder. Wilson y Wilder, en la sesentena entonces, se conocían desde finales de los años veinte, cuando el joven crítico modernista había defendido de los ataques marxistas a un joven novelista de fama incipiente, que se había dado a conocer con la novela The Cabala (La cábala 1926) y había ganado su primer premio Pulitzer por The Bridge of San Luis Rey (El puente de San Luis Rey, 1927), sorprendiendo después al mundo internacional de lectores con la elegancia exquisita y el poder de evocación de The Woman of Andros (La mujer de Andros, 1930) y Heaven, my Destination (Mi destino, el cielo, 1935) -una serie de novelas en las que Wilson había descubierto complacido la rápida influencia de Marcel Proust-, y que empezaba, tras años de experimentaciones formales, a estudiar con seriedad las posibilidades de la escritura dramática².

Treinta años después, Wilder era ya un autor consagrado por el público internacional, con otros dos premios Pulitzer en su haber por las obras teatrales Our Town (Nuestra ciudad, 1938) y The Skin of Our Teeth (Por un pelo, 1942), y consideraba a Wilson, como le había advertido escrupulosamente a Kanin antes de la visita, el crítico literario vivo más destacado de su generación ${ }^{3}$. Al inicio de los años sesenta, sin embargo, la lucha de clases literaria que Wilson había mantenido en solitario, con su peculiar interpretación histórica de la literatura, se había difuminado y el ambiente, según Kanin -casi veinte años más joven que el resto del grupo reunido aquella tarde--, parecía de otra época y lugar, tal vez más propio de quienes se recuperaban del trauma del reconocimiento de una literatura, como la americana, en la que la crítica había aparecido simultáneamente a la creación y con la misma potencia en casi todos los géneros (el teatro había tenido que esperar a la llegada de Eugene O'Neill), sin que hubiera dado tiempo a la elaboración de una distinción y una recepción adecuadas, ni académica ni socialmente. De hecho, Wilson se había quejado en el inicio de su carrera -despejando así su propio camino- de la inexistencia de críticos puros ante la obra de creadores demasiado conscientes de sus facultades y capaces de una comunicación central con sus lectores, al menos desde los trascendentalistas del siglo $\mathrm{XIX}^{4}$.

\footnotetext{
${ }^{1}$ Este texto forma parte de la Introducción a Thornton Wilder, Nuestra ciudad, edición y traducción de A. Lastra y J.A. Peris-Cancio, Biblioteca del Siglo XX, Cátedra, Madrid, 2020 (en curso de publicación). Agradezco a la editorial el permiso para su reproducción, así como al profesor Peris-Cancio una colaboración de la que he aprendido mucho.

${ }^{2}$ The Angel that Troubled the Water and Other Plays (El ángel que turbó las aguas y otras obras teatrales) apareció en 1928; The Long Christmas Dinner and Other Plays in One Act (La larga cena de Navidad y otras obras teatrales de un acto) en 1931. Se trataba en conjunto de veintidós piezas breves en las que Wilder ensayaría su concepción de la puesta en escena despojada de decorados y del presente perpetuo de la narración dramática. Wilder había empezado a escribir pequeñas composiciones teatrales a los quince años. Las dos series, así como las obras de teatro no publicadas por Wilder, se han reunido en Collected Plays and WritingsonTheater (2007). Las cinco primeras novelas de Wilder se han reunido en The Bridge of San Luis Rey and Other Novels 1926-1948 (2009).

${ }^{3}$ Wilson había tenido que defender también a Wilder de la acusación de haber plagiado en Por un pelo la novela de James Joyce Finnegans Wake, publicada en 1939. Wilder esbozó, con el espíritu de Alexander Pope, una carta de respuesta a los críticos Joseph Campbell y Henry Morton Robinson que decidió no enviar al final, en la que decía que "la industria, propia de hormigas, de los pedantes, reuniendo fragmentos aislados, ha confundido la naturaleza de la influencia literaria desde que los primeros críticos empezaron a considerar los libros una rama de la mercancía en lugar de una expresión de energía" (Wilder, 2008: 414).

${ }^{4}$ No es difícil trazar una línea desde Ralph Waldo Emerson hasta Gertrude Stein, que pasa por William James y se ramifica extraordinariamente en un sentido que conserva siempre la pedagogía de la transmisión: al terminar la Guerra Civil, Emerson fundó el primer departamento de Filosofía de los Estados Unidos, del que William James sería el primer gran profesor y en el que Stein se convirtió en la mejor alumna de James. Wilder, por su parte, se consideraba un discípulo de Stein. La tendencia a la soledad de Wilder es claramente thoreauviana-aunque fue muy crítico con los trascendentalistas en general - y parte de la geografía de Nuestra ciudad (aun siendo "geografía" un término de Stein) resulta familiar para el lector de Walden, cuyo capítulo central se llamaba "The Village" (La pequeña ciudad). El mayor logro de Wilson como crítico fue inspirar The Library of America, cuyas ediciones han elaborado el canon real de la literatura americana. En la actualidad comprende tres volúmenes dedicados a Wilder.
} 
La conversación fluía en torno a Francis Scott Fitzgerald hasta que el nombre de Maugham suscitó de repente un violento ataque de Wilson, cuyos argumentos, en líneas generales, se remontaban a su reseña de la novela histórica de Maugham Then and Now (Entonces y ahora), publicada en 1946, con el añadido de que, según los postulados de la ética literaria de Wilson, el escritor y su obra estaban inextricablemente unidos y resultaba imposible separarlos: toda crítica ad opus equivalía a una crítica ad hominem. Kanin defendió a su amigo ausente, pero la conclusión de Wilson sobre el "escritor vulgar que escribe mal, pero está patrocinado por lectores no demasiado serios que no se preocupan por la escritura", tenía que desarmar a Kanin si no podía persuadirse del todo de que su admiración personal por el autor no era mayor que el gusto por sus obras y, por tanto, reafirmaba su convicción, tan ligera como forzada por las circunstancias, de que la crítica de Wilson se debía, en realidad, a que el señor Maugham había invadido el terreno de la crítica literaria, un terreno que le estaría vedado como autor solo porque Wilson había fracasado relativamente en el terreno de la ficción. La envidia es siempre una suposición y conduce, antes o después, a la calumnia, que obra en todas las direcciones posibles 5 .

Sin embargo, que Kanin hiciera pasar por una conversación privada tan improvisada como real lo que Wilson había publicado hacía casi dos décadas podría parecer casi una corroboración de las opiniones de Wilson sobre el fraude del señor Maugham y el tipo de escritor que, a su juicio, simulaba ser ante el público que él mismo había generado (los "trescientos millones de ejemplares" que Maugham había vendido) si no fuera por la presencia, en casa de Wilson, de Wilder. Esa noche, Kanin trató de "repasar con Thornton el drama vivido por la tarde", pero Wilder prefirió mantenerse neutral. Quería, le contestó, meditarlo y pensarlo detenidamente. En su opinión, muchos críticos defendían la idea de que "si algo se vende bien es sospechoso", y el autor de Clásicos y comerciales parecía encajar a la perfección en el grupo. En opinión de esos críticos, escribir debía ser únicamente la distracción del caballero, cuyo deber consistía en instruir, entretener o edificar a sus semejantes, lo que excluía ganar dinero con ello y, por tanto, la profesión literaria como tal, aunque pudiera suscitar lo que a menudo se ha considerado, como si se tratara de una categoría estética suficiente, la vergüenza del éxito que lleva a un escritor a ocultar, debajo de una superficie convencional, la profundidad de sus intenciones o, como el propio Wilson había escrito de Wilder, a creer que cualquier libro que alcance la popularidad esconde entre líneas alguna forma más o menos inconfesable de sentimentalismo y falsedad.

Con esta perspectiva, podía pensarse que tanto Maugham, el viejo escritor europeo, heredero de mil y un relatos, como Wilder, el viejo escritor americano que había tenido que apoderarse de una cultura por su propio esfuerzo, habían hecho dejación de responsabilidad de sus obras, al incorporarse a un mercado global, a favor del crítico literario, que estaba en condiciones de entenderlos mucho mejor de lo que ellos se entendían a sí mismos. En última instancia, el crítico representaba la conciencia de clase literaria. No por casualidad Wilder había sido el centro de la mayor polémica literaria de la época: ser acusado de superficial no era muy distinto de disfrutar parasitariamente de un lugar en la superestructura social ${ }^{6}$.

\section{La escritura dramática}

La indecisión de Wilder aquella noche es significativa. Kanin y Ruth Gordon eran amigos suyos desde hacía casi tanto tiempo como Wilson, en la infinita extensión de las relaciones sociales de Wilder, y su amistad había discurrido en paralelo a su carrera teatral (Gordon había interpretado en 1938 el papel de Norah en la adaptación de Casa de muñecas de Ibsen que Wilder había llevado a cabo mientras escribía Nuestra ciudad), pero en el fondo estaba de acuerdo con Wilson en que su forma de entender la literatura no podía tener mucho que ver con la del señor Maugham, por "comerciales" que también hubieran resultado sus

\footnotetext{
${ }^{5}$ Véase Garson Kanin (1966). Wilder es el autor más citado en el libro después del propio Maugham. El trasfondo de la conversación registrada por Kanin se encuentra en la crítica literaria de Wilson. Véanse, sobre todo, sus libros The Shores of Light. A Literary Chronicle of the Twenties and Thirties (1952; que contiene su recepción de las primeras novelas de Wilder y la polémica con el crítico marxista Michael Gold), y Classics and Commercials. A Literary Chronicle of the Forties (1950; que contiene la defensa de Por un pelo de Wilder en el contexto de la aparición de Finnegans Wake, así como el artículo "The Apotheosis of Somerset Maugham", de 1946, de donde Kanin copia literalmente, sin citarlo, todo el planteamiento de la discusión sobre Maugham). "Lucha de clases literaria", "interpretación histórica de la literatura" y "trauma del reconocimiento" son frases características de la crítica de Wilson.

${ }^{6}$ En The Alcestiad (A/cestíada, 1955), Wilder exploraría el subtema del trabajo de Apolo para Admeto.
} 
obras y -lo que seguramente habría sido motivo de una reserva más acentuada por su parte- por mucho que nadie discutiera entonces (ni ahora) su condición de "clásico". Sin duda, la escritura dramática le había ofrecido a Wilder, a diferencia de la escritura novelística que seguiría cultivando hasta el final de su vida7, la justificación para ser tan convencional como hiciera falta: el teatro era, en sus propias palabras, "una mentira permitida" y, por comparación con la novela, tenía una espontaneidad, derivada del presente perpetuo en el que los personajes se encontraban sobre la escena, que se interponía deliberadamente entre el narrador y el público, de manera que el escritor adquiría una fuerza adicional a la fuerza de la que se había prevalido durante siglos en forma de "coro, prólogo, epílogo o raisonneur" y que solo era parangonable a la que empezaba a tener (como Wilder había observado en su colaboración con Alfred Hitchcock) el director cinematográfico, gracias, en parte, a que el cine estaba abandonando aceleradamente las nociones de tiempo y lugar heredadas del teatro e incluso la aspiración a la originalidad artística, arriesgándose a correr el "peligro teorético" de quedar por debajo de la literatura. Invisible, el punto de vista del dramaturgo acababa imponiéndose a la atención del espectador. Que el propio Wilder se hiciera visible como actor en numerosas ocasiones, encarnando al Director de Escena de Nuestra ciudad o al señor Antrobus de Por un pelo (evocando así a Shakespeare en el papel del Espectro de Hamlet o en el del viejo Adam en As you like it [Como gustéis]), lejos de contradecir esa estrategia narrativa, la reforzaba. Todos los intentos por explicar la obra de Wilder mediante referencias autobiográficas o personales olvidan que la principal función de la convención teatral -y es muy difícil ahora leer sus novelas de otra manera que como borradores escénicos o guiones cinematográficos - es la de elevar lo específico a lo general. Preguntarse por el propio éxito de Wilder no tenía, por ello, una respuesta sencilla siguiendo los esquemas marxistas, que Wilson entendía perfectamente, aunque no los compartiera hasta el punto de sacrificar a Wilder en el altar del realismo social y la revolución, pero tampoco siguiendo los esquemas despolitizados y sensacionalistas en los que se basaba la veneración de Kanin por Maugham. Los críticos que han empezado a estudiar la relación de Wilder con Bertolt Brecht y el distanciamiento teatral tropiezan una y otra vez con la ausencia de una crítica adecuada de la obra de Wilder. En cualquier caso, Wilson no era Walter Benjamin ${ }^{8}$.

Wilder tenía razón en que el teatro era la más convencional de las artes. Lo que a sus contemporáneos podía parecerles novedoso en la puesta en escena de sus obras no lo era para quienes, como el propio Wilder, tenían una mirada de conjunto de la fenomenología teatral, desde la tragedia y la comedia antiguas - que Wilder recrearía en su A/cestíada y en el examen de Edipo rey de Sófocles- hasta las representaciones del Siglo de Oro español, especialmente el teatro de Lope de Vega que Wilder estudiaría durante años, además de adaptar a Henrik Ibsen y haber asistido en Roma al estreno de Seis personajes en busca de autor de Luigi Pirandello. En cualquier caso, no podría parecerle novedoso a nadie con el paso del tiempo ${ }^{9}$. Al autor le correspondía sostener, en aras de la coherencia y para salvar las apariencias, el desarrollo de una idea, lo que Wilder - tomando prestado el término clave de Gertrude Stein- Ilamaría "narración". A diferencia de Maugham, cuya reputación, en medio de las vanguardias artísticas, residía en su adhesión a la gran tradición de la literatura inglesa, en la que un escritor siempre podía encontrar un repertorio paralelo de ficciones que respondían más o menos satisfactoriamente al problema del realismo en la literatura -era demasiado conocida al respecto, y comprensible solo desde la posición que ocupaba, su hostilidad hacia las últimas e inclasificables obras de Henry James, que tanto Wilson como Stein elevaron por encima de casi cualquier otra forma de escritura hasta la aparición de Joyce-, Wilder tenía que hacer frente a una variante del problema del realismo que, de diversas formas, la literatura americana obligaba a plantear: el envejecimiento prematuro de todo cuanto en América resultaba nuevo, pero inalcanzable. No hacían falta los esquemas marxistas para darse cuenta de que ese sería el problema de la literatura en la época de la globalización y Wilder lo captó muy bien al interpretar - en la que probablemente habría sido su mayor contribución dramática-El castillo de Franz Kafka como un "emporio". Maugham podía resultar exótico y sofisticado en su recreación de los confines del Imperio británico - desde Malasia hasta las alcobas de la aristocracia-, pero no tenía que enfrentarse al drama de "la vida de una pequeña ciudad frente a la vida de las estrellas", como escribió Wilder en el primer prefacio a Nuestra ciudad, un drama que consistía en

\footnotetext{
${ }^{7}$ The Eight Day (El octavo día) se publicó en 1967; Theophilus North en 1973. Véase Wilder (2011).

${ }^{8}$ Véase Wilson Pereira de Oliveira (2010). Véase también, en el terreno de la novela, Antonio Cascón Dorado (2010). The Idus of March (Los Idus de Marzo) de Wilder se publicó en 1948.

9 En 2003, Paul Newman protagonizaría una producción de teatro cinematográfico para televisión de Nuestra ciudad (disponible en la red) fiel a los planteamientos de Wilder, aunque conservando algunos de los viejos vicios de la primera producción de Jed Harris, que, para el espectador, resulta innecesariamente nostálgica.
} 
"encontrar un valor por encima de cualquier precio para los acontecimientos más pequeños de nuestra vida diaria", como escribiría, veinte años después, en el segundo prefacio ${ }^{10}$.

Entre los dos prefacios se encuentra la historia efectiva de Nuestra ciudad, que comprende tanto su ideación (Wilder escribió la mayor parte de la obra en el extranjero y llevaría ese desplazamiento hasta situar Gorver's Corners, "nuestra ciudad", con unas coordenadas precisas, en medio del océano, entre otras inexactitudes que deslocalizaban la comunidad) ${ }^{11}$, como su producción teatral, que en cierto modo desvirtuaría una representación despojada de todo escenario y desnuda de sentimentalismo, y la posterior adaptación al medio cinematográfico y su recuperación para la lectura individual, que inevitablemente pasaba por la traducción a otras lenguas. En la serie de conferencias que Wilder impartiría en Harvard tras la Segunda Guerra Mundial con el propósito de describir las "características americanas", el paso del lenguaje americano a la soledad americana evocaba ante su auditorio la dificultad de ser americano en los términos en los que Wilder había llegado a entender esa dificultad: el problema de la soledad americana - que ya era la soledad moderna por antonomasia, nuestra soledad- era el problema de la "pertenencia". Wilder sabía que pertenecía a lo que Emily Dickinson, a quien dedicaba la más penetrante de sus conferencias, había llamado "inmensidad" y que, además, pertenecía a esa inmensidad como un artista que reconocía al mismo tiempo "el orden, los límites, las premisas tácitas compartidas y las convenciones"; pero, como Dickinson, Wilder se había adentrado desde el principio en nuevos territorios de la escritura, percibiendo con tanta prontitud como delicadeza las manifestaciones espirituales que se iban propagando, y no parecía llegado el momento de consolidar lo que había adquirido, marcar esos límites y establecer esas convenciones. "Aquellos que viven en la inmensidad no son críticos literarios melindrosos" -escribió- ni probablemente los necesiten ${ }^{12}$.

\section{Narración}

La narración de Nuestra ciudad está orientada hacia esa inmensidad y la dirección de la escritura le fue marcada por el menos melindroso de los críticos y escritores americanos. Hasta qué punto Nuestra ciudad depende de las enseñanzas de Gertrude Stein, a quien Wilder se refería como su "Diótima", es una de las entradas más difíciles, y más compensatorias, de los estudios sobre Wilder. Se ha hablado de influencia y de catálisis, de anticipación y coincidencia. Wilder había ensayado en muchas de sus obras en un solo acto previas a Nuestra ciudad la renuncia a la trama y la acción dramática, así como el despojamiento del escenario - como había hecho Stein en sus propias obras de teatro-, pero aún había de encontrar la forma definitiva para lo que quería decir. Diez años después de El puente de San Luis Rey y a pesar de que seguía trabajando en El cielo, mi destino, esa forma definitiva tenía que permitirle desaparecer como autor de novelas para que la vida cotidiana, sobre la que no cabe la omnisciencia, ocupara el lugar que le correspondía. El 13 de septiembre de 1937, Wilder escribió a Stein que ya no podía ocultarle que estaba escribiendo "la más hermosa de las obritas que puedas imaginar [...] Una pequeña obra con todos los

\footnotetext{
${ }^{10}$ Véanse las notas de trabajo y las escenas que Wilder llegó a esbozar de The Emporium en The Journals of Thornton Wilder 1939-1961 (1985:295-336), y en Collected Plays and Writings onTheater(2007: 462-495). Wilder pensó en presentar la obra como una "sucesión de prólogos". El tema era la "inadecuación de lo ético" frente a "la solidez del emporio". Wilder anotó que la primera impresión de K. es que el castillo se parece a la ciudad (town) en la que había nacido y que el castillo también era la Ley. "Veo que esta obra que tomó como punto de partida El castillo de Kafka se mueve cada vez más hacia modalidades de Finnegans Wake. Tal vez esta obra tenga originalidad, sea original. Siempre he tenido claro que las otras dos [Nuestra ciudad y Por un pelo] no lo eran. Las otras dos eran calqués. Puede que ahora haya digerido profundamente mi posesión de los conceptos de tiempo, mis conceptos de la situación humana, y sean tan permeables que me pueda permitir escribir una obra verdaderamente original, original no en el sentido de que esté llena de recursos nuevos, sino de que haga ver a la gente por primera vez cosas que hasta ahora sabía sin ser conscientes de que las sabía" (Wilder, 1985: 326).

11 "Hablamos la última vez de lo difícil que es ser americano. Nos referimos al apoyo que los europeos reciben de todos esos elementos que llamamos entorno: lugar, tradición. costumbres (Yo soy porque mis vecinos me conocen). Su entorno está tan espesamente tejido, resulta tan sólido, que el muchacho y la muchacha que están creciendo tienen algo contra lo que toparse. El americano, por otra parte, está en el mar, desconectado del lugar, sin confianza en la autoridad, arrojado a sí mismo" ("The American Loneliness", 1952, en Wilder, 1979: 35).

${ }^{12}$ Véase Wilder ("American Characteristics [Towards an American Language, The American Loneliness, Emily Dickinson]", 1952: 3-63, en Wilder, 1979: 60).
} 
grandes temas y una gran obra con todas las pequeñas cosas de la vida encantadoramente impresas", y añadía a continuación:

Esta obra es una inmersión, una inmersión en una ciudad de New Hampshire. Se llama Nuestra ciudady su tercer acto se basa en tus ideas como en grandes pilares y, lo sepas o no, hasta nuevo aviso, ya estamos colaborando estrechamente (en Burns et al., 1996: 174-175).

La carta no se conocería en vida de Wilder y es el único documento explícito de su deuda con Stein. En público, Wilder solía explicar que el último acto se basaba en el Purgatorio de Dante y era obvia la inspiración de la Spoon River Anthology (Antología de SpoonRiver, 1915) de Edgar Lee Masters. Pero las "ideas" de Stein habían sido liberadoras para Wilder. Es significativo que Wilder y Stein se conocieran en un ambiente académico, en el que ambos eran unos extraños que demostraron ser excelentes maestros, y que la Universidad de Chicago, donde Wilder era profesor invitado, publicara con prólogo de Wilder las lecciones de Stein. Wilder observaría que esas lecciones eran en sí mismas modelos de forma artística. La "repetición" - uno de los recursos más célebres de Stein- estaba al servicio de la vida, la historia y la naturaleza, y exigía la colaboración del lector. Al mismo tiempo, las lecciones de Stein se convertían en objeto del método de enseñanza: "No se aprende nada si no es en respuesta a una pregunta profundamente clavada y planteada con claridad". La pregunta por la narración suscitaba una enseñanza semejante y, en la respuesta de Stein, la vida de Nuestra ciudadempezaba a insinuarse: "Narración es lo que cualquiera tiene que decir de cualquier modo sobre cualquier cosa que pueda suceder, que haya sucedido o vaya a suceder de cualquier modo". No es difícil darse cuenta de que el Director de Escena de Nuestra ciudad, que Wilder encarnaría con frecuencia, "repetía" las ideas de Stein: la "narración" tenía que ver con la relación entre el artista y el público-con lo que ambos tenían que decirse- $y$, sobre todo, con la creación de un lenguaje característicamente americano que se contrapusiera a una soledad tan americana como humana.

En el prólogo a The Geographical History of America (Historia geográfica de América, 1936), Wilder recuerda la diferencia que Stein establecía entre "naturaleza humana" y "mente humana"; la primera tenía que ver con la identidad y requería la presencia de un público; la segunda era la "pura existencia" y el público era una amenaza para ella: "En su expresión más elevada [la mente humana] ni siquiera es un público para sí misma". La condición de los Muertos en el Acto III de Nuestra ciudad tiene que ver con la mente humana, mientras los vivos siguen aferrados a su naturaleza:

Si nadie tuviera que morir — citaba Wilder a Stein — no habría sitio suficiente para que ninguno de nosotros que ahora vive hubiera vivido. No habríamos sido si todos los demás no hubieran muerto. No habría habido sitio.

Ahora bien, la relación de la naturaleza humana con la mente humana es esta.

La naturaleza humana no lo sabe...

Pero la mente humana puede...

El comentario de Wilder podría figurar como acotación escénica a Nuestra ciudad. "La naturaleza humana, aferrándose a la supervivencia de la identidad, no puede darse cuenta de una situación sin identidad. La mente humana, que no conoce el tiempo ni la identidad en sí, puede darse cuenta de esto como un hecho objetivo de la experiencia". Aunque todos los espectadores de Nuestra ciudad, en la experiencia común del teatro, y naturalmente todos los lectores en su experiencia privada, se han emocionado genuinamente en el Acto III, Wilder descubre que el sentimiento adecuado -el sentimiento adecuado en el que consiste la enseñanza de Stein- es la alegría producida por la irrupción de la vida cotidiana, sin la cual no podrían plantearse las grandes preguntas ${ }^{13}$.

Esas grandes preguntas no son patrimonio del escritor ni del crítico. La "geografía" de Stein tiene que ver con la localización de la naturaleza humana. Wilder extrajo la más profunda de las enseñanzas de Stein al darse cuenta de que los Estados Unidos, independientes de la geografía tanto como de la historia, "prometen producir una civilización en la que la mente humana no solo aparezca en una obra maestra

\footnotetext{
${ }^{13}$ Wilder era un excelente lector de filosofía, de Alfred North Whitehead a José Ortega y Gasset (a quien sirvió de intérprete en 1949 en los encuentros de Aspen sobre Goethe), y habría apreciado la contraposición de Hans Blumenberg (2007) entre "tiempo de la vida" y "tiempo del mundo" (Lebenszeit y Weltzeit) como una aproximación adecuada a la "inmensidad" de Nuestra ciudad.
} 
ocasional, sino que en muchos aspectos se distribuya por toda la gente". Wilder llamaría a esa distribución la cultura en una democracia.

En 1947, Wilder publicó el último de sus escritos sobre Stein, que había muerto el año anterior. Nuestra ciudad se había convertido en una pieza habitual en los teatros americanos y, tras la guerra, en casi todos los escenarios del mundo - la representaron los partisanos yugoslavos y los marines americanos en Japón, se representó en la Alemania y en la Italia de la posguerra, empezó a traducirse a casi todas las lenguas y es una tradición en los institutos de enseñanza secundaria de los Estados Unidos-, y Wilder había pasado por la experiencia de Joyce. Pero la evocación de Stein es la más profunda y poderosa de las interpretaciones de sí mismo y de las aspiraciones de Nuestra ciudad. Four in America (Cuatro en América) "está lleno de esa extrañeza que debe provenir del don poético para devolver la intensidad a las imágenes cubiertas por el polvo de la costumbre y la rutina". Para recuperar esa intensidad es preciso escuchar. Wilder llamó a Stein an impassioned listener to life. Nosotros podemos llamarlo así a él14.

Ochenta años después de su estreno, Nuestra ciudad sigue necesitando que el lector escuche lo que la vida tiene que decirle. Que Wilder le concediera la preeminencia a la escucha es una advertencia para su porvenir. En la mañana del 22 de enero de 1938, apenas unas horas antes del preestreno de Nuestra ciudad en el Teatro McCarter de Princeton, Wilder escribió una nota sobre los aspectos de la producción y la dirección de la obra que Jed Harris había preparado. En esa nota le reprochaba al escenógrafo el "sentido sorprendentemente débil de la reconstrucción visual"15. ¿Escuchamos la vida viendo una obra de teatro o leyéndola? Buena parte de las obras de teatro y de las novelas de Wilder se han adaptado al cine desde la temprana versión de El puente de San Luis Rey en 1929 y Nuestra ciudad no es una excepción. Más allá de su colaboración con Hitchcok en el guion de The Shadow of a Doubt (La sombra de una duda, 1943), Wilder parece haber perdido el gusto por el cine, pero es incierto si, con la mirada puesta en el futuro desde el cementerio de Hamden en Connecticut, donde reposa como uno de sus muertos, con el tono tranquilo y sin melancolía, vería una repetición o reconstrucción visual de su ciudad en la obra teatral Middletown (2010) de Will Eno - por sinceramente conmovedora que resulte- o más bien en la desolada puesta en escena de la película de Lars von TrierDogville (2003). ¿Corre la cámara el "peligro teorético", e incluso el peligro real, de quedar por debajo de la literatura en su intento de resolver el problema de la realidad?

\section{Referencias bibliográficas}

Blumenberg, H. (2007): Tiempo de la vida y tiempo del mundo. Valencia: Pre-Textos. [Trad. de M. Canet].

Burns, E., Dydo, U.E. y Rice, W. eds. (1996): The Letters of Gertrude Stein and Thornton Wilder. New Haven: Yale University Press.

Cascón Dorado, A. (2010): "Tres autores en busca del personaje Julio César: Wilder, Brecht, Warner", en Moreno, A. ed.: Julio César: textos, contextos y recepción. De la Roma clásica al mundo actual. Madrid: UNED.

Kanin, G. (1966): Remembering Mr. Maugham. Nueva York: Atheneum. [Trad. Recordando al Sr. Maugham, prólogo de Noël Coward, trad. de S. Carral, Hatari! Books, Madrid, 2018: 222-226]

Pereira de Oliveira, W. (2010): Brecht visita Nossa Cidade, de Thornton Wilder: aproximações e distanciamentos. Belo Horizonte: Escola de Belas Artes/UFGM. Disponible en web: http://www.bibliotecadigital.ufmg.br/dspace/handle/1843/JSS959JKK?show=full.

Wilder, T. (1979): American Characteristics and Other Essays. New York: Harper \&Row. [Ed. de D. Gallup].

- (1985): The Journals of Thornton Wilder 1939-1961. New Haven: Yale UniversityPress. [Ed. de D. Gallup].

-(2007): Collected Plays and Writings onTheater. Nueva York: The Library ofAmerica. [Ed. de J.D. McClatchy].

- (2008): The Selected Letters of Thornton Wilder. Nueva York: Harper Collins. [Ed. de R.G. Wilder y J.R. Bryer].

-(2009): The Bridge of San Luis Rey and Other Novels 1926-1948. Nueva York: The Library ofAmerica. [Ed. de J.D. McClatchy]

—(2011): The Eighth Day, Theophilus North, Autobiographical Writings. Nueva York: The Library ofAmerica. [ed. de J.D. McClatchy].

\footnotetext{
${ }^{14}$ Los prólogos de Wilder se han reunido en American Characteristics and OtherEssays (1979: 181-221).

${ }^{15}$ Véase el prontuario de Wilder en TheLettersof Gertrude Stein and Thornton Wilder (Burns et al., 1996: 375-376).
} 
Wilson, E. (1950): Classics and Commercials. A Literary Chronicle of the Forties. Nueva York: Farrar, Strauss and Company.

- (1952): The Shores of Light. A Literary Chronicle of the Twenties and Thirties. Nueva York: Farrar, Straus, Giroux.

\section{Breve CV del autor}

Antonio Lastra (Valencia, España, 1967) es doctor en Filosofía, profesor de Filosofía en la Educación Secundaria, investigador externo del Instituto Franklin de Investigación en Pensamiento Norteamericano de la Universidad de Alcalá, investigador del Instituto Universitario de Investigación en Filosofía Edith Stein de la Universidad Católica de Valencia y profesor de Centro Universitario en la Escuela de Empresarios de Valencia (EDEM). Preside La Torre del Virrey. Instituto de Estudios Culturales Avanzados y dirige la Escuela de Filosofía del Ateneo de Valencia. Sus campos de trabajo preferentes son la ecología de la cultura, la traducción como lingua franca, la escritura constitucional americana, el problema teológico-político, la literatura inglesa y los estudios sobre cine. Su último libro es Aprender leyendo (Ápeiron Ediciones, Madrid, 2018). 